\title{
De-Centered Central Stars of Planetary Nebulae
}

\author{
Aubrie McLean, Martín A. Guerrero, Robert A. Gruendl, \& You-Hua \\ $\mathrm{Chu}$ \\ Department of Astronomy, University of Illinois at Urbana-Champaign, \\ 1002 W. Green St., Urbana, IL 61801, USA
}

\begin{abstract}
The origin of the wide range of morphologies observed in planetary nebulae $(\mathrm{PNe})$ is not well established. The influence of a binary companion of the central star can naturally explain this variety of morphologies, but very few PNe have known binary central stars. The evolution of the binary system with mass loss may result in the displacement of the central star from the nebular center. The large sample of $\mathrm{PNe}$ observed by HST is being used to search for de-centered central stars. Preliminary results indicate that the occurrence of de-centered central stars is widespread among all morphological types of PNe.
\end{abstract}

\section{De-Centered Central Stars of Planetary Nebulae}

Most $\mathrm{PNe}$ are aspherical and show elliptical, bipolar, multi-polar, or pointsymmetric morphologies, and many others show a rich variety of highly collimated structures, such as FLIERs and jet-like features. The origin of asphericity in $\mathrm{PNe}$ is controversial. The influence of binary companions is often invoked to explain the asphericity of PNe. The direct detection of binary companions to the central stars of PNe, however, is difficult (Bond 2000).

It has been suggested that the evolution of the central star of a $\mathrm{PN}$ in a binary system will cause it to migrate from the center of the PN as a result of the peculiar velocity component of the ejected material introduced by the orbital motion of the progenitor star (Soker \& Rappaport 2001). This is an easily observable effect that may provide an indirect probe of binarity.

The detection and measurement of the offset of the central star of a PN from the center of the PN requires high-spatial resolution because the expected offset is small, and a sharp image of the nebula is needed to determine its center. The large number of high-quality WFPC2 images of PNe in the $H S T$ archive is an invaluable dataset that can be used to measure the offset of central stars from the center of symmetry of PNe.

Previous studies of $H S T$ images for a few individual bipolar and pointsymmetric PNe have revealed the off-center position of their central stars, e.g., MyCn 18 and NGC 6884 (Sahai 1999; Miranda et al. 1999)(Fig. 1). This has strengthened the interpretation that de-centered central stars of PNe provide evidence of binary systems and that such systems play an important role in the shaping of highly aspherical PNe. 

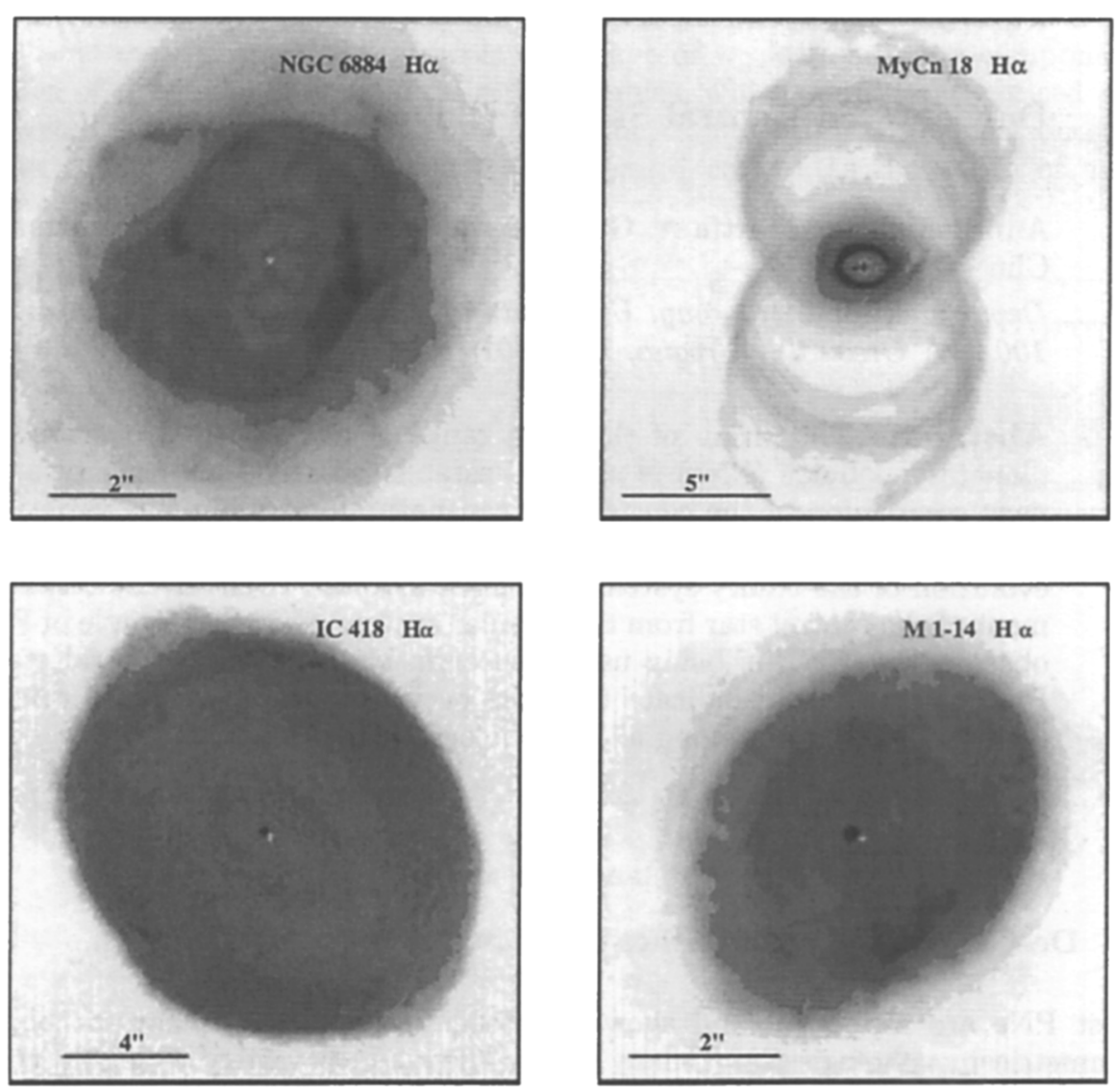

Figure 1. HST $\mathrm{H} \alpha$ images of $\mathrm{PNe}$ with de-centered central stars: (top) NGC 6884 (point-symmetric) and MyCn 18 (bipolar), and (bottom) IC 418 and M 1-14 (elliptical). The position of the center of symmetry is marked by a cross.

We have an on-going program to study the detailed symmetry of the large sample of PNe observed by $H S T$ and to measure the offset of the central stars from the symmetry center. Preliminary results show that elliptical PNe also host de-centered central stars (e.g., IC 418 and M 1-14)(Fig. 1), thus de-centered central stars are found among all morphological types of PNe. Clearly, a statistical correlation between the morphology of $\mathrm{PNe}$ and the existence and amount of the offset or its absence is needed before a definite interpretation can be reached.

\section{References}

Bond, H. E. 2000, Asymmetrical Planetary Nebulae II, 115

Miranda, L., Guerrero, M. A., \& Torrelles, J. M. 1999, AJ, 117, 1421

Sahai, R. et al. 1999, AJ, 118, 468

Soker, N., \& Rappaport, R. 2001, ApJ, 557, 256 